Paediatr Paedolog 2017 · 52:125

DOI 10.1007/s00608-017-0495-8

Online publiziert: 27. Juli 2017

๑) Springer-Verlag GmbH Austria 2017

CrossMark

Hochwertige Lehre und Ausbildung sind Grundvoraussetzungen für eine optimale Versorgungsqualität im medizinischen Bereich. Ebenso besteht einhelliger Konsens hinsichtlich der Sinnhaftigkeit von lebenslanger bzw. lebensbegleitender Weiter- und Fortbildung. Auch im Bologna-Prozess, einem visionären Projekt mit dem Ziel, die Hochschulausbildung in Europa - mittlerweile haben sich 47 Staaten diesem Prozess angeschlossen - im Sinn eines europäischen Hochschulraums vergleichbar zu machen und $\mathrm{zu}$ vereinheitlichen, wird lebenslangem Lernen berufsgruppenunabhängig ein entsprechend hoher Stellenwert eingeräumt. Der Erfordernis, die Wissensbasis ständig zu verbreitern und zu vertiefen, trägt die seit September 2016 gesetzlich verpflichtende aktive Dokumentation ärztlicher Fortbildungen in standardisierter, nachvollziehbarer, international vergleichbarer Form Rechnung.

\section{Unüberblickbare Informationsflut}

Eine zunehmende Verschulung und Überregulierung von Prozessen, die einen Wissenszuwachs zum Ziel haben, die mittlerweile fast unüberblickbare Informations- und Datenflut und die ungeheure Dichte an Fortbildungsveranstaltungen erleichtern den Prozess aktiver ständiger Wissenserweiterung jedoch nicht. Fragen wie „Was ist für unser alltägliches ärztliches Handeln praktisch relevant?“ oder „Von welcher im Zuge einer Fortbildungsveranstaltung erworbenen Information hat (auch) mein Patient, meine Patientin einen unmittelbaren Nutzen?" sind oftmals unbeantwortbar. Unter diesen Aspekten ist die Programmgestaltung von Tagungen und Fortbildungsveranstaltungen

\title{
Martin Benesch
}

Univ.-Klinik für Kinder- und Jugendheilkunde, Medizinische Universität Graz, Graz, Österreich

\section{Lebenslanges Lernen}

für die Organisatoren eine besondere Herausforderung.

\section{Jahrestagung der ÖGKJ}

Die Jahrestagungen der Österreichischen Gesellschaft für Kinder- und Jugendheilkunde (ÖGKJ) verbinden traditionellerweise praxis- und versorgungsrelevante Inhalte mit innovativen und gesundheitspolitisch aktuellen Themen. Unter dem Motto „Kinder wachsen - Kind erwachsen" wollen wir bei der 55. Jahrestagung die Rolle der Pädiatrie für die Gesundheit im Erwachsenenalter herausstreichen und die spezifischen Unterschiede der verschiedenen Phasen der Kindheit bis zum Erwachsenwerden beleuchten.

Der Stellenwert unserer Jahrestagung als Plattform für den interdisziplinären Wissens- und Informationsaustausch wird auch durch die Teilnahme der Österreichischen Gesellschaft für Prä- und Perinatale Medizin und der Österreichischen Gesellschaft für Kinder- und Jugendchirurgie unterstrichen, worüber wir uns sehr freuen. Gleichzeitig werden wir dem wissenschaftlichen Nachwuchs die Gelegenheit geben, ihre Forschungsergebnisse einem breiten Fachpublikum vorzustellen. Am Mittwoch, den 20.09.2017, findet ein Präsymposium mit einer Reihe von Workshops und Seminaren statt, in denen praktische Fertigkeiten auf dem neuesten Wissensstand vermittelt werden. Nicht unerwähnt lassen möchte ich den gesellschaftlichkommunikativen Wert unserer Jahrestagungen. Abseits von beruflichem Alltag haben wir die Gelegenheit, Kolleginnen und Kollegen in einer entspannten Atmosphäre zu treffen, sich untereinander auszutauschen und Kontakte aufzufrischen.

\section{pädiatrie \& pädologie Kongressausgabe}

Bezüglich inhaltlicher Schwerpunkte und Highlights der Tagung verweise ich auf das Interview in dieser Ausgabe, das pädiatrie \& pädologie mit dem Präsidenten der 55. Jahrestagung, Herrn Univ.Prof. Dr. Christian Urban, Leiter der Univ.-Klinik für Kinder- und Jugendheilkunde der Medizinischen Universität Graz geführt hat.

\section{Danksagung}

An dieser Stelle möchte ich mich für die freundschaftliche und unkomplizierte Zusammenarbeit mit allen an der Organisation dieser Jahrestagung Beteiligten, insbesondere beim Tagungspräsidenten und bei der Tagungssekretärin Frau OÄ Dr. Jasmin Pansy von der Klinischen Abteilung für Neonatologie unseres Hauses, bei den Gutachtern der Abstracts, die hier namentlich natürlich nicht genannt werden können, und bei Herrn Ralph Kerschbaumer und seinem Team ganz herzlich bedanken.

\section{Korrespondenzadresse}

Univ.-Prof. Dr. M. Benesch
Univ.-Klinik für Kinder-
und Jugendheilkunde,
Medizinische Universität Graz
Auenbruggerplatz 38,
8036 Graz, Österreich
martin.benesch@klinikum-
graz.at
$\begin{aligned} & \text { Kinder- Univ.-Klinik für } \\ & \text { Jugendheilkunde } \\ & \text { Graz }\end{aligned}$

Univ.-Prof. Dr. Martin Benesch, Tagungssekretär der 55. Jahrestagung der Österreichischen Gesellschaft für Kinder- und Jugendheilkunde

Interessenkonflikt. M. Benesch gibt an, dass kein Interessenkonflikt besteht. 